\title{
Sequential plan-view imaging of sub-surface structures in the transmission electron microscope
}

F.C-P. Massabuau ${ }^{1,2}$, H.P. Springbett ${ }^{1}$, G. Divitini ${ }^{1}$, P.H. Griffin ${ }^{1}$, T. Zhu ${ }^{1}$, R.A. Oliver ${ }^{1}$

${ }^{1}$ Department of Materials Science and Metallurgy, University of Cambridge, Cambridge CB3 OFS, UK

${ }^{2}$ Department of Physics, SUPA, University of Strathclyde, Glasgow G4 ONG, UK

E-mail: f.massabuau@strath.ac.uk

\section{Keywords}

Transmission electron microscopy, Sample preparation, Layered structures, Gallium Nitride, Dislocations

\begin{abstract}
Transmission electron microscopy (TEM) is a central technique for the characterisation of materials at the atomic scale. However, it requires the sample to be thin enough to be electron transparent, imposing strict limitations when studying thick structures in plan-view. Here we present a method for sequential plan-view TEM that allows one to image complex structures at various depths. The approach consists of performing an iterative series of front-side ion milling followed by TEM imaging. We show it is possible to image how the sample properties vary with depth up to several microns below the surface, with no degradation of the sample and imaging conditions throughout the experiment. We apply this approach to 3D cavities in mesoporous GaN distributed Bragg reflectors, demonstrating the ability to characterise the morphology of the pores, local crystal features and chemical composition through the multilayer structure. The same workflow can be applied to a variety of complex micron-scale systems which are by nature too thick for standard TEM analysis, and can also be adapted for profiling samples in cross-section.
\end{abstract}


Thin films are the building blocks of a wide variety of devices, including light emitting diodes [1], solar cells [2], transistors [3, 4], magnetic field sensors [5], thermoelectric coolers [6, 7], and triboelectric nanogenerators [8]. To understand the physical principles underpinning operation it is often necessary to investigate the local properties of the material with high spatial resolution through the entire thickness of the device. To this effect, transmission electron microscopy (TEM) is routinely used as it allows to observe the atomic structure of defects [9-13], compositional fluctuations of alloys [13-17], phase and orientation of crystallites [18-20], luminescence properties across a sample [21-23], dopant distribution in semiconductor devices [24, 25], magnetic field orientation and strength [26, 27], amongst many others.

Only a minority of samples - such as nanomaterials (e.g. nanoparticles, nanowires) - can be readily observed in the TEM in their native state. For the vast majority of materials and devices, sample preparation is not only necessary, it is instrumental in the acquisition of good quality TEM data - and is perhaps as important a step as the imaging itself. TEM sample preparation often requires a lot of practice from the user, and uncontrolled preparation conditions could lead to serious data misinterpretation. For example, focussed ion beam (FIB) sample preparation using inappropriate milling conditions can cause amorphization [28, 29], phase change [30], or generation of dislocations [31] in the material. Even under perfectly controlled conditions, specific reactions and stresses induced into the sample can make a TEM specimen non-representative. For example, hydrogen was found to be incorporated into Ti samples prepared using conventional room temperature FIB (using Ga ion or Xe plasma source) or electropolishing [32]. As another example, dissociated dislocations were found in $\mathrm{GaN}$ foils as a result of strain relaxation occurring during mechanical sample preparation [33].

There are many ways to prepare TEM foils - mechanical polishing, FIB, electropolishing, ultramicrotomy, to cite only a few [34] - and the appropriate method will depend on the material properties (e.g. mechanical, electrical, chemical). The only immovable condition is geometrical: the sample must be electron-transparent in the direction of observation. The required thickness will depend on the sample itself (e.g. density) as well as on the microscope settings (e.g. acceleration voltage). As a rule of thumb, if one wants to properly image a sample in the TEM, the region of interest must be thinner than $200 \mathrm{~nm}$ - and much thinner if high resolution imaging or electron energy loss spectroscopy is required.

For plan-view imaging, often required for analysing crystal defects or observing e.g. chemical inhomogeneities in the deposition plane (the plane parallel to substrate), standard preparation methods involve mechanical polishing followed by ion polishing. In this configuration the sample is thinned down from the back (i.e. from the substrate), and the electron transparency condition implies that it is not possible to image the material properties deeper than $c a .200 \mathrm{~nm}$ below the top surface of the sample (illustrated in Figure 1(b)(top)). This is however a serious limitation for many samples (e.g. where 
several layers are stacked) for which the (structural, chemical, luminescence, etc.) properties vary with depth. In this paper we present a TEM sample preparation and data acquisition workflow for sequential plan-view imaging. We demonstrate the capabilities of the approach with the study of the pore morphology in a cavity structure which utilises mesoporous GaN distributed Bragg reflectors (DBR). This sample is technologically relevant for blue single photon emission and displays advantages over standard non-porous structures (principally in terms of background emission) [35]. The sample consists of a stack of forty $c a .50 \mathrm{~nm}$ thick layers of alternating porous and non-porous $\mathrm{GaN}$, with a $c a .200 \mathrm{~nm}$ non-porous GaN cavity in the middle of the stack and which encloses a thin InGaN quantum dot layer. To add more structural variations with depth, the porosification parameters have been deliberately changed between the top half and bottom half of the structure. Detailed description of the sample structure is provided in the "Growth and etching" section. Conventional TEM preparation and investigation on this sample would have been insufficient to characterise the full range of pore morphologies through the multilayer. The stack of layers, $c a .2 .5 \mu \mathrm{m}$ in thickness, is well above the maximum thickness for TEM investigation, and conventional plan-view imaging would only provide information on a very limited part of the device. Other standard approaches would be also be suboptimal; cross-sectional imaging would only provide limited information on the pore structure along the growth direction; and tomography approaches (both electron tomography [36-38] and atom-probe tomography [39-41]), which present the advantage of 3D imagery, would not access a sufficiently large volume to provide statistically relevant information. 
(a)

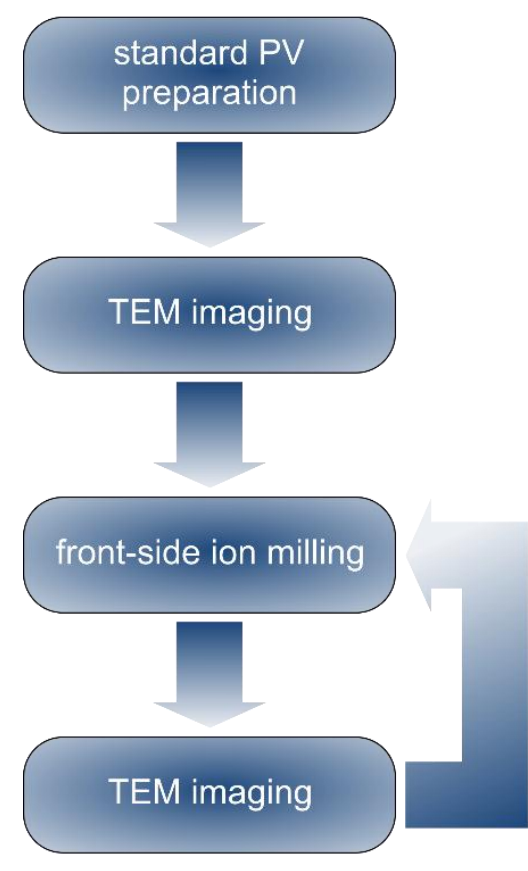

(b)

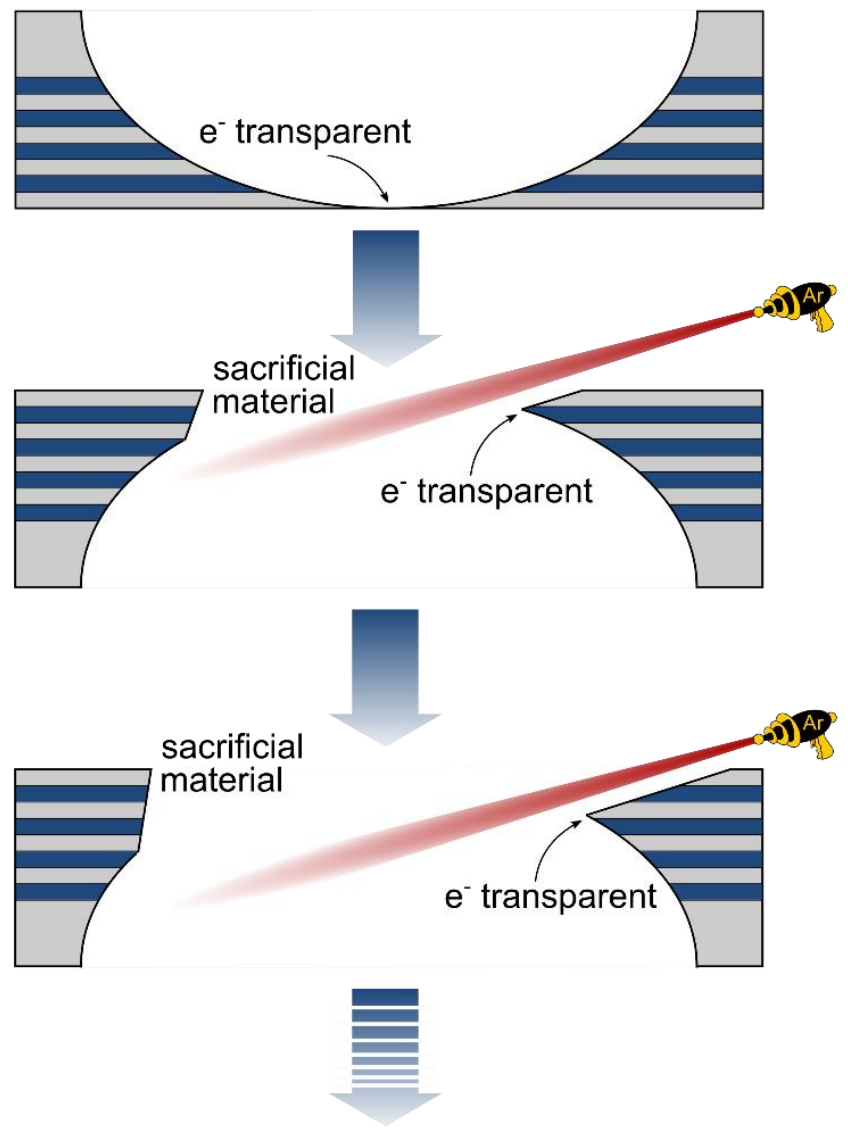

(c)

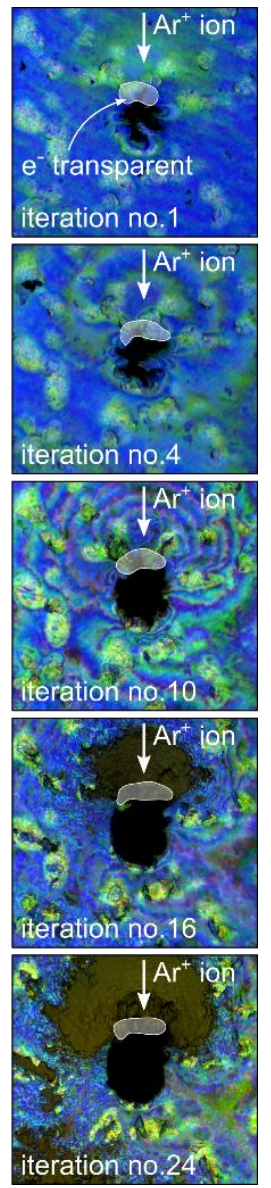

Figure $1-(a-b)$ Schematic of the sequential plan-view TEM methodology. (c) Optical images of the sample taken on the ion polishing system showing how the morphology of the hole evolves with consecutive iterations (the shaded region indicates the region of interest for TEM imaging).

The sequential plan-view TEM procedure is as follow, the principle is illustrated in Figure 1:

1. Standard plan-view preparation. The sample is first prepared for plan-view imaging using the standard preparation technique. In this study, the sample was mechanically ground from the backside (i.e. substrate side) using $\mathrm{SiC}$ and diamond grinding disks until the sample reached a thickness of $c a .50 \mu \mathrm{m}$. A dimpler with a cotton wheel and diamond polishing paste ( $1 \mu \mathrm{m}$ particle size $)$ was then used to remove scratches from grinding. Final thinning of the sample was conducted using an $\mathrm{Ar}^{+}$ion mill in a Gatan PIPS II system. The ion guns were operated without modulation (i.e. the guns constantly on while the sample is rotated) with a top incidence angle of $5^{\circ}$. It is important to keep the angle as shallow as possible in anticipation for the front-side milling in step 3 . The beam energy was kept at $5 \mathrm{keV}$ until thickness fringes were visible in the sample, then the energy was lowered to $3 \mathrm{keV}$ until a hole formed in the sample. A final cleaning step was performed at $1 \mathrm{keV}$ for 10 minutes and $0.1 \mathrm{keV}$ for 10 minutes. 
2. TEM imaging. The sample is observed in the TEM, here using high-angle annular dark field scanning TEM (HAADF-STEM). Given that the hole formed at the top surface of the sample, only the very top < $200 \mathrm{~nm}$ of material can be observed - as illustrated in Figure 1(b)(top).

3. Front-side ion milling. After TEM analysis, the sample is mounted in the ion mill system with the front-side exposed to the ion gun. Because a hole has now formed, it is impossible to use the ion guns without modulation as this would lead to blunting the edges of the hole (these must be kept as thin as possible to allow imaging). Instead, the ion mill was operated in single modulation (i.e. the guns are on only when the same side of the rotating sample is exposed): this results in removal of material from the top surface on one side of the hole and sacrificing the other side of the hole - as illustrated in Figure 1(b)(middle). The incidence angle was $5^{\circ}$, which results in an overall angle of the TEM foil next to the thinned hole of $10^{\circ}\left(5^{\circ}\right.$ from the back-side milling from step 1 and $5^{\circ}$ from the front-side milling) - this is why using shallow angles is important in order to maintain a sufficiently wide electron transparent field of view at each step. Since this step aims for the gentle removal of a limited amount of material, the guns were operated at $1 \mathrm{keV}$ for $\mathrm{X}$ minutes, and 0.1 $\mathrm{keV}$ for 10 minutes - where $\mathrm{X}$ is chosen to remove the appropriate amount of material (empirically determined based on the material itself as well as the targeted thickness to remove). In this experiment, we found that 5 minutes was adequate provided the thickness of the individual layers $(c a .50 \mathrm{~nm}$ ) but it was found that the milling time had to be increased to 7 minutes later on in the experiment (iteration no. >15-20) to compensate for the increase in hole size which resulted in a slower milling rate. Further discussion and guidance regarding the milling duration are given in the "Limitations and recommendations" section.

4. TEM imaging. TEM analysis of the sample is then carried out. The region of interest is now the material on the side of the hole (shaded regions in Figure 1(c)) which has undergone thinning and therefore allows to observe the material deeper within the sample - as illustrated in Figure 1(b)(middle, bottom).

5. Steps 3 and 4 are repeated as many times as required, adjusting the duration of the $1 \mathrm{keV}$ ion milling as a way of controlling the depth. It is crucial to perform all the front-side milling iterations (step 3) at the same position and same sample orientation so that the same parts of the hole are thinned and the same parts are sacrificed. For this study, we conducted 23 iterations of step 3 and 4 - Figure 1(c) shows an optical image of the evolution of the hole throughout the experiment (iteration no.1 corresponds to the standard plan-view preparation, step 1). A montage of TEM images acquired throughout the experiment is given in Supplementary Information (Figure S1). 

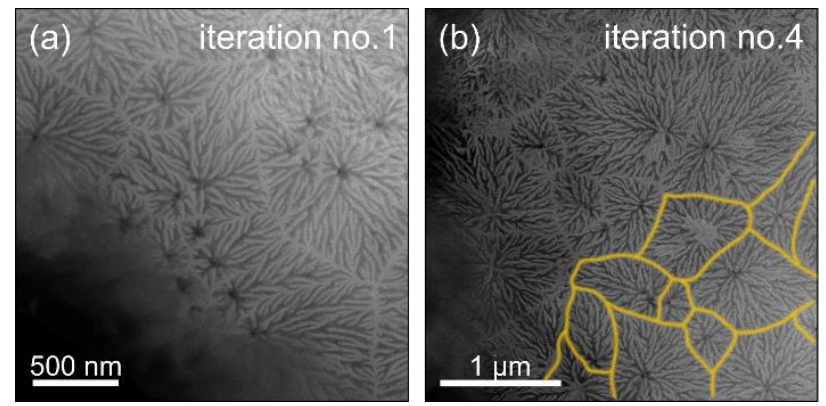

Figure 2. HAADF-STEM images of the porous GaN layers reached at iterations no. (a) 1, and (b) 4. Here contrast is thickness-dependent.

Figure 2 gives an overview of the morphology of the porous layers - in this instance near the top surface of the sample (as indicated by the low iteration no.). In these images, since the layers are only made of $\mathrm{GaN}$ the contrast is given by the thickness of the foil, i.e. pores appear dark and non-pores appear bright. We can see that the morphology of the porous layers consists of several pore domains separated by nonporous walls (a few are highlighted in yellow in Figure 2(b)). Each pore domain originates from a black dot in the images, which is in fact a hollow channel from an etched dislocation (exemplified in Figure 6(a)). This is consistent with previous literature which showed that dislocations act as channels for porosification of the material [42]. What our new sample preparation approach reveals is how the pore domain structure evolves with depth through the multilayer, as we will highlight in the following paragraphs.
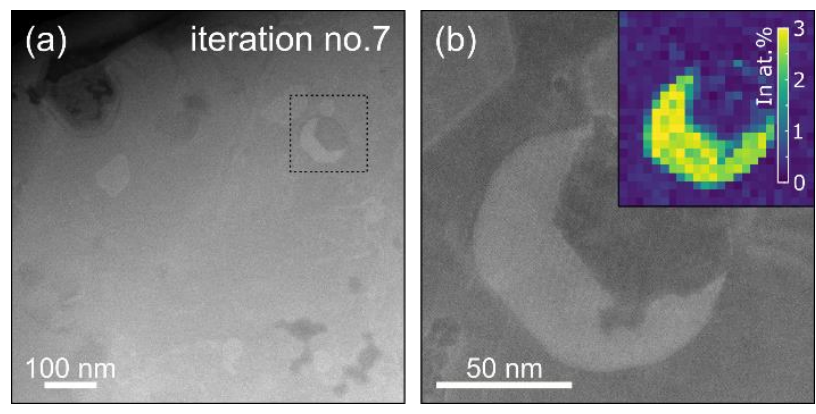

Figure 3. HAADF-STEM images of the InGaN quantum dot layer reached at iteration no. 7. Image (b) corresponds to a magnified region indicated by a square in (a). Here contrast is mainly composition-dependent. In inset, map of indium distribution by EDX.

Figure 3 shows that it was possible to observe the InGaN quantum dot layer after 6 iterations of frontside ion milling (step 3), that is, at iteration no. 7. This provides a strong evidence that the sample preparation technique presented here is effective. The sample we used is almost entirely made of GaN apart from the InGaN quantum dot layer which is located $c a .1 \mu \mathrm{m}$ below the surface. Evidence of the presence of indium is provided by the energy dispersive X-ray spectroscopy (EDX) map in inset. Locating and imaging this specific layer would have been impossible using a standard sample preparation methodology as it is located too deep inside the stack. 

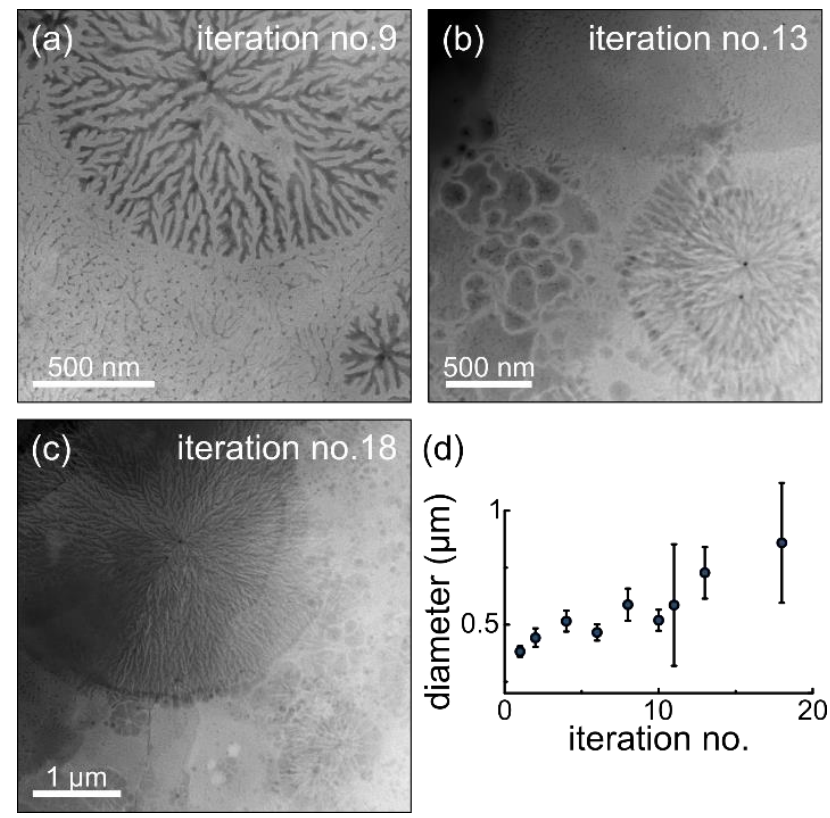

Figure 4. HAADF-STEM images of the porous GaN layers reached at iterations no. (a) 9, (b) 13, and (c) 18. Here contrast is thickness-dependent. (d) Summary plot of the evolution of the size of the pore domains with iteration no. (i.e. depth through the structure).

Figure 4 highlights the morphology of the layers located below the InGaN quantum dot layer - i.e. iteration no. >7. The morphology in the bottom DBR (Figure 4) contains pore domains of a similar nature to the ones observed in the top DBR (Figure 2). However, as can be seen visually throughout Figure 2 and 4(a-c), as well as in the plot in Figure 4(d), the size of the domains increases the deeper the porous layer is located, reaching several micrometres in size for the bottom-most layers. Additionally Figure 4(a-c) shows that, past the InGaN quantum dot layer, other morphologies appear outside of the pore domains - a few more detailed micrographs of these regions are shown in Figure 5. 

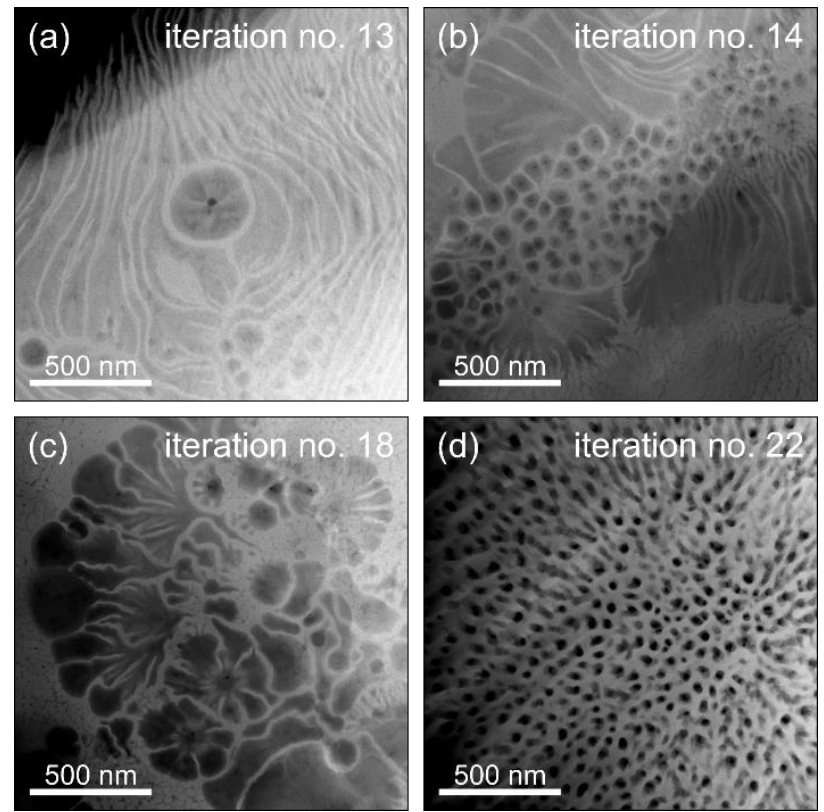

Figure 5. HAADF-STEM images obtained from the layers located deeper than the InGaN quantum dot layer (iteration no. >7). Here contrast is thickness-dependent.

Figure 5 highlights some alternative porous structures that were observed in the bottom DBR. While the top DBR exhibits a morphology that is solely dictated by pore domains originating from dislocation channels (as in Figure 2), the bottom DBR exhibits alternative morphologies, on top of some remaining enlarged pore domains shown in Figure 4. We expect that these are induced in part by the deliberate change in parameters during the porosification process of the bottom DBRs, although other factors may have contributed to the observed structure, such as, the greater distance of the layer to the top surface (i.e. to the etching solution reservoir) which may favour a competition between different porosification processes. While the understanding of formation mechanism of these complex structures is beyond the scope of this work, the observation of a variety of morphologies shows the flexibility of this approach, as well as demonstrating how sub-surface analysis is able to recover information that cannot be anticipated from conventional plan-view imaging of the top layer alone.
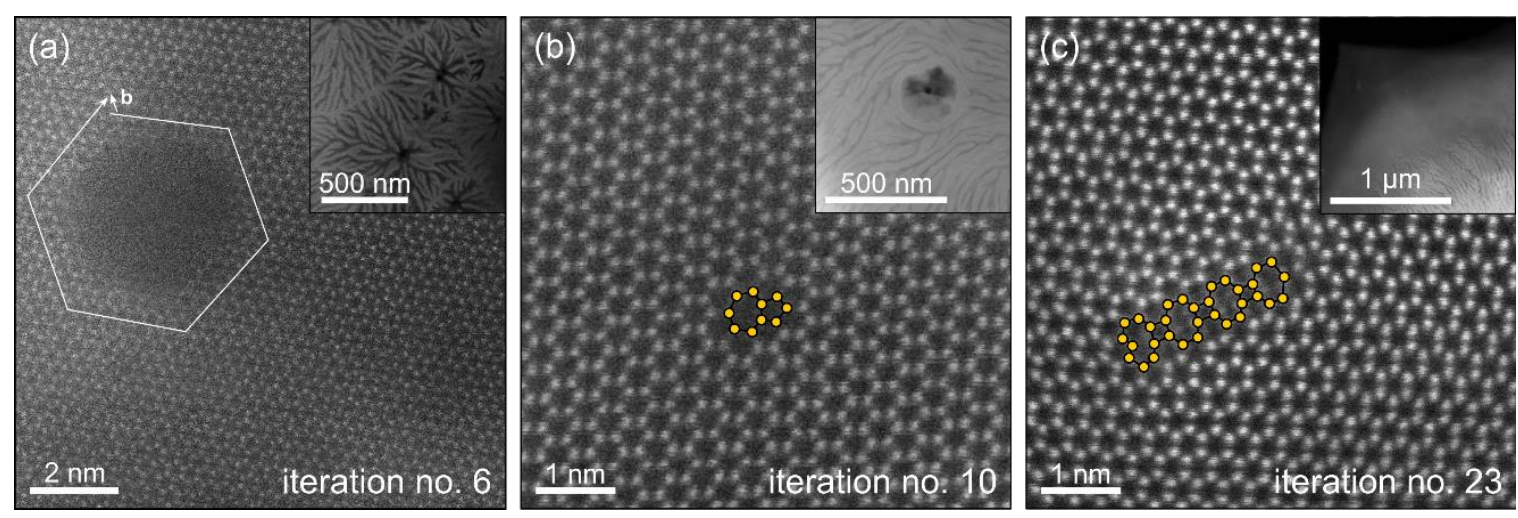

Figure 6. High-resolution STEM images of (a) a dislocation channel at the centre of a pore domain obtained at iteration no. 6, (b) an unetched edge-type dislocation (5/7 atom ring core) obtained at iteration no. 10, (c) an unetched dissociated mixed-type dislocation (9/4/8/4/8/4/7 atom ring core) in a 

HAADF-STEM images.

One point that must be emphasised is that, despite several iterations of ion milling, the sample remains of excellent quality, because of the use of low voltages for the front-side ion milling steps. This allowed atomic resolution imaging - e.g. allowing dislocation core analysis, determination of Burgers circuit near holes - to be achieved throughout the whole experiment. This includes late stages of the experiment such as iteration no. 23 (e.g. Figure 6(c)) for which we estimate that the sample has undergone overall more than 6 hours of front-side ion milling to expose the layers $c a$. 2-2.5 $\mu \mathrm{m}$ below the sample surface. A few examples of atomic resolution images obtained at different stage of the experiment are given in Figure 6. For instance, we could confirm previous reports indicating that the pore domains originate from nanometre size channels etched at dislocations - the (open) Burgers circuit in Figure 6(a) betrays the presence of a dislocation [42]. In places, we could find some dislocations which were left unetched, in the porous layer (Figure 6(b)) or in the non-porous layer (Figure 6(c)). The core structure could be identified and is in agreement with core configurations commonly found in III-Nitride materials [12, 13].

In conclusion, we designed a plan-view TEM sample preparation method that allows the user to overcome the sample thickness limitation imposed by traditional sample preparation. By using iterative front-side ion milling and TEM imaging, it is possible to observe how the plan-view structure of a sample evolves up to several microns below the surface. The procedure is gentle enough to keep the sample suited for sensitive analysis (e.g. atomic resolution imaging) throughout the experiment. We demonstrated the methodology on a mesoporous GaN cavity structure with an InGaN quantum dot active region to highlight the different pore morphologies throughout the DBR. The methodology applies in theory to any complex structures which are too thick to be fully observed using standard sample preparation techniques. Lastly the methodology could also be adapted for profiling samples in cross-section.

\section{Limitation and recommendations}

Identifying the appropriate duration for front-side ion milling. Step 3 is central to the experiment, and it is thus critical to have reasonable control over the ion milling conditions to avoid "blind observations" of the sample. The milling rate of the sample depends on the material being milled as well as on the settings of the ion mill itself (e.g. current, beam focus, stage rotation). The appropriate amount of material to remove between each iteration is also experiment-dependent. Therefore prior to undertaking the sequential plan-view TEM experiment, the user must have a reasonable estimate of the time required to mill the sample. To facilitate this, we make the following recommendations:

- Ideal procedure: A test sample consisting of the dominant material in the sample to analyse, and containing easily identifiable markers at a specific depth (typically a few 100s nm) below 
the surface should be prepared prior to the main experiment. This pre-experiment would not necessarily require a full preparation for TEM analysis (Step 1-2): the bulk sample could just undergo top-surface ion milling and be checked regularly in a (preferably low voltage) scanning electron microscope (SEM) until the markers are detected.

- Minimal procedure: The experimenter should have minimal knowledge of the sample structure (layers and their approximate thickness) as well as of the behaviour of the sample or similar in the ion mill - for example through previous conventional TEM sample preparations. In the present manuscript, the authors had ample experience with conventional sample preparation of GaN materials, hence that option was sufficient to achieve the desired results.

Studying samples containing voids. Samples that contain voids should be analysed carefully as it should be kept in mind that the incidence angle of the ion beam during milling will be different on the main material and on the void's wall due to geometric effects. This may lead to uneven milling rates, higher for the walls that experience quasi-perpendicular ion beam incidence compared to the rest of the sample that experiences grazing incidence milling. The effect of a heterogeneous milling rate could result in data misinterpretations. Furthermore, the iterative ion milling steps can amplify this effect and associated misinterpretations. In the present experiment, this effect was not observed as we could see similar pore morphologies in related samples that did not undergo ion milling (e.g. diagonally cleaved samples observed in the SEM) or in samples that underwent different ion milling geometries [43]. However, we cannot rule out that this will be significant for some samples. We thus recommend operating a few trial observations using a preparation that involves no or very low amount of milling.

\section{Growth and etching}

The as-grown sample (i.e. before etching) consists of two periodic structures made of alternating GaN and n-doped GaN layers, with an InGaN quantum dot layer between the two structures (Figure 7(left)). The sample was grown by metal-organic vapour phase epitaxy in a 6 x 2 inch Thomas Swan closecoupled showerhead reactor on a $c$-plane sapphire substrate. Trimethylgallium, ammonia and silane were used as $\mathrm{Ga}, \mathrm{N}$ and Si precursors, respectively, and hydrogen as a carrier gas. A $4 \mu \mathrm{m}$ thick GaN buffer layer with a nominal dislocation density of $c a .3 \times 10^{8} \mathrm{~cm}^{-2}$ was first grown, followed by a 500 $\mathrm{nm}$ n-doped GaN (silicon doping concentration $c a .1 \times 10^{18} \mathrm{~cm}^{-3}$ ). The first periodic structure was then deposited as 10 pairs of alternating highly doped n-GaN (47 nm thick) and undoped GaN (56 nm thick) layers. The InGaN quantum dot layer was grown using a modified droplet epitaxy method [44], and was located in the centre of a $200 \mathrm{~nm}$ thick undoped GaN layer. The second periodic structure is then deposited identically to the first.

The sample was then electrochemically etched to transform the n-doped layers into porous $\mathrm{GaN}$, following the process described in Ref. [45]. This process transforms the periodic structures into DBRs. The surface of the as-grown sample was connected to a Solartron 1287A potentiostat and immersed in 
a $0.25 \mathrm{M}$ oxalic acid electrolyte. A Pt foil was used as the cathode, and the sample was used as the anode. Upon application of a potential bias between the anode and cathode, the conductive $\mathrm{n}$-GaN layers were porosified (Figure 7(right)). To add more variation to the pore morphology throughout the structure, the potentiostat settings were changed for the etching of the bottom DBR. A schematic of the sample structure is shown in Figure 7. As can be seen the total thickness of the structure approaches 2.5 $\mu \mathrm{m}$.

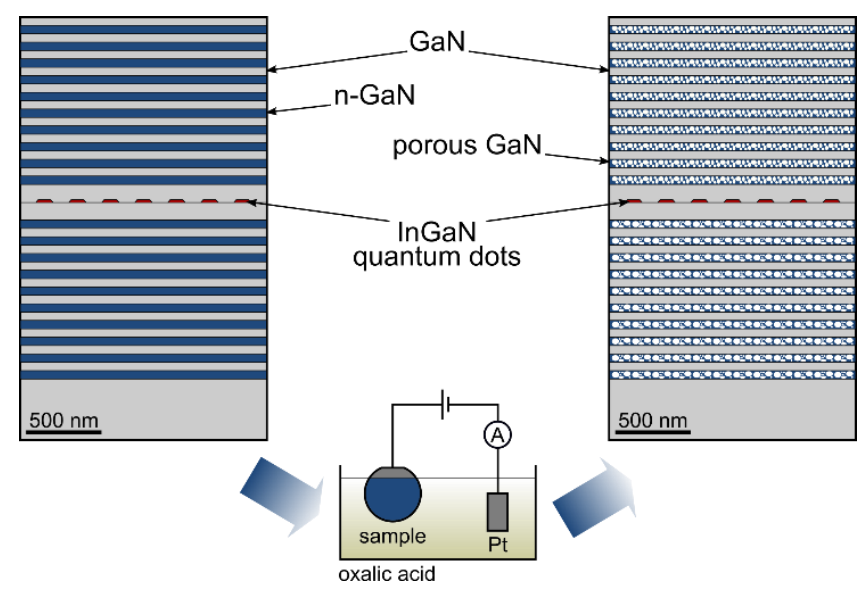

Figure 7- Schematic of the sample structure before (left) and after (right) etching. Schematic of the etching experimental setup in the middle.

\section{Transmission electron microscopy}

The sample was observed in plan-view using a FEI Titan ${ }^{3}$ aberration-corrected scanning transmission electron microscope (STEM) operated at $300 \mathrm{kV}$ in high angle annular dark field STEM (HAADFSTEM) mode. Energy dispersive X-ray spectroscopy (EDS) was conducted on a FEI Osiris at $200 \mathrm{kV}$. Quantification was obtained using the Cliff-Lorimer factor method [46].

\section{Acknowledgement}

This work was supported in part by the UK Engineering and Physical Sciences Research Council (EPSRC) under Grant Nos. EP/M011682/1 and EP/M010589/1 and the EPSRC Impact Acceleration Account Follow On Fund of the University of Cambridge. PG would like to acknowledge funding from $\mathrm{EP} / \mathrm{L} 015455 / 1$.

\section{Author contribution}

F.M. designed the experiment. F.M., H.S. and G.D. conducted the TEM sample preparation and acquired the data. H.S. analysed the data. T.Z. provided the initial material, and P.G. etched the sample. F.M., G.D. and R.O. prepared the manuscript. All authors discussed, revised, and approved the manuscript.

\section{Competing interests}


The authors declare no competing interests.

\section{Materials and correspondence}

E-mail: f.massabuau@strath.ac.uk

\section{References}

[1] S. Pimputkar, J.S. Speck, S.P. DenBaars, and S. Nakamura, Nat. Photon. 3, 180 (2009)

[2] M. Liu, M.B. Johnston, and H.J. Snaith, Nature 501, 395 (2013)

[3] H. Amano et al., J. Phys. D: Appl. Phys. 51, 163001 (2018)

[4] A.F. Paterson, and T.D. Anthopoulos, Nat. Comm. 9, 5264 (2018)

[5] M.N. Baibich, J.M. Broto, A. Fert, F. Nguyen Van Dau, F. Petroff, P. Eitenne, G. Creuzet, A. Friederich, and J. Chazelasm, Phys. Rev. Lett. 61, 2472 (1988)

[6] I. Chowdhury, R. Prasher, K. Lofgreen, G. Chrysler, S. Narasimhan, R. Mahajan, D. Koester, R. Alley, and R. Venkatasubramanian, Nat. Nano. 4, 235 (2009)

[7] W. Jin, L. Liu, T. Yang, H. Shen, J. Zhu, W. Xu, S. Li, Q. Li, L. Chi, C. Di, and D. Zhu, Nat. Comm. 9, 3586 (2018)

[8] F. Fan, Z-Q. TianZhong, and L. Wang, Nano Energy 1, 328 (2012)

[9] S. Kondo, A. Ishihara, E. Tochigi, N. Shibata, and Y. Ikuhara, Nat. Comm. 10, 2112 (2019)

[10] H. Yang, J.G. Lozano, T.J. Pennycook, L. Jones, P.B. Hirsch, and P.D. Nellist, Nat. Comm. 6, $7266(2015)$

[11] A.H. Heuer, C.L. Jia, and K.P.D. Lagerlöf, Science 330, 1227 (2010)

[12] S.K. Rhode, M.K. Horton, M.J. Kappers, S. Zhang, C. J. Humphreys, R.O. Dusane, S.-L. Sahonta, and M.A. Moram, Phys. Rev. Lett. 111, 025502 (2013)

[13] F.C-P. Massabuau, S.L. Rhode, M.K. Horton, T.J. O’Hanlon, A. Kovács, M.S. Zielinski, M.J. Kappers, R.E. Dunin-Borkowski, C.J. Humphreys, and R.A. Oliver, Nano Lett. 17, 4846 (2017)

[14] M.K. Horton, S. Rhode, S.-L. Sahonta, M.J. Kappers, S.J. Haigh, T.J. Pennycook, C.J. Humphreys, R.O. Dusane, and M.A. Moram, Nano Lett. 15, 923 (2015)

[15] A.R. Bowman, M.T. Klug, T.A.S. Doherty, M.D. Farrar, S.P. Senanayak, B. Wenger, G. Divitini, E.P. Booker, Z. Andaji-Garmaroudi, S. Macpherson, E. Ruggeri, H. Sirringhaus, H.J. Snaith, and S.D. Stranks, ACS Energy Lett. 4, 2301 (2019) 
[16] K. Pantzas, G. Patriarche, D. Troadec, M. Kociak, N. Cherkashin, M. Hÿtch, J. Barjon, C. Tanguy, T. Rivera, S. Suresh, and A. Ougazzaden, J. Appl. Phys. 117, 055705 (2015)

[17] Q. Ding, Y Zhang, X. Chen, X. Fu, D. Chen, S. Chen, L. Gu, F. Wei, H. Bei, Y. Gao, M. Wen, J. Li, Z. Zhang, T. Zhu, R.O. Ritchie, and Q. Yu, Nature 574, 223 (2019)

[18] J.K. Sunde, D.N. Johnstone, S. Wenner, A.T.J. van Helvoort, P.A. Midgley, and R. Holmestad, Acta Materiala 166, 584 (2019)

[19] J.W. Roberts, J.C. Jarman, D.N. Johnstone, P.A. Midgley, P.R. Chalker, R.A. Oliver, and F.CP. Massabuau, J. Cryst. Growth 487, 23 (2018)

[20] J. Guo, G. Haberfehlner, J. Rosalie, L. Li, M.J. Duarte, G. Kothleitner, G. Dehm, Y. He, R. Pippan, and Z. Zhang, Nat. Comm. 9, 946 (2018)

[21] R. Bourrellier, S. Meuret, A. Tararan, O. Stéphan, M. Kociak, L.H.G. Tizei, and A. Zobelli, Nano Lett. 16, 4317 (2016)

[22] J.T. Griffiths, S. Zhang, B. Rouet-Leduc, W.Y. Fu, A. Bao, D. Zhu, D.J. Wallis, A. Howkins, I. Boyd, D. Stowe, M.J. Kappers, C.J. Humphreys, and R.A. Oliver, Nano Letters 15, 7639 (2015)

[23] M. Muller, F. Bertram, P. Veit, B. Loitsch, J. Winnerl, S. Matich, J.J. Finley, G. Koblmuller, and J. Christen, Appl. Phys. Lett. 115, 243102 (2019)

[24] D. Cooper, J. Phys. D: Appl. Phys. 49, 474001 (2016)

[25] D. Avram, C. Colbea, M. Florea, S. Lazar,D. Stroppac, and C. Tiseanu, Nanoscale 11, 16743 (2019)

[26] C. Jin, Z-A. Li, A. Kovacs, J. Caron, F. Zheng, F.N. Rybakov, N.S. Kiselev, H. Du, S. Blugel, M. Tian, Y. Zhang, M. Farle, and R.E. Dunin-Borkowski, Nat. Comm. 8, 15569 (2017)

[27] J.C. Loudon, A.C. Twitchett-Harrison, D. Cortés-Ortuño, M.T. Birch, L.A. Turnbull, A. Stefancic, F.Y. Ogrin, E.O. Burgos-Parra, N. Bukin, A. Laurenson, H. Popescu, M. Beg, O. Hovorka, H. Fangohr, P.A. Midgley, G. Balakrishnan, and P.D. Hatton, Adv. Mater. 31, 1806589 (2019)

[28] M. Schaffer, B. Schaffer, and Q. Ramasse, Ultramicroscopy 114, 62 (2012)

[29] J. Mayer, L.A. Giannuzzi, T. Kamino, and J. Michael, MRS Bulletin 32, 400 (2007)

[30] R. Prasath Babu, S. Irukuvarghula, A. Harte, and M. Preuss, Acta Materiala 120, 391 (2016)

[31] M. Andrzejczuk, T. Plocinski, W. Zielinski, and K.J. Kurzydlowski, J. Microsc. 237, 439 (2010) 
[32] Y. Chang, W. Lu, J. Guénolé, L.T. Stephenson, A. Szczpaniak, P. Kontis, A.K. Ackerman, F.F. Dear, I. Mouton, X. Zhong, S. Zhang, D. Dye, C.H. Liebscher, D. Ponge, S. Korte-Kerzel, D. Raabe, and B. Gault, Nat. Comm. 10, 942 (2019)

[33] I. Bellabas, I.G. Vasileiadis, J. Moneta, J. Smalc-Koziorowska, and G.P. Dimitrakopoulos, J. Appl. Phys. 126, 165702 (2019)

[34] D.B. Williams, and C.B. Carter, Transmission electron microscopy: a textbook for materials science, 2nd ed. New York : Springer (2009)

[35] H.P. Springbett, K. Gao, J. Jarman, T. Zhu, M. Holmes, Y. Arakawa, and R.A. Oliver, Appl. Phys. Lett. 113, 101107 (2018)

[36] D. Kong, H. Wang, J.J. Cha, M. Pasta, K.J. Koski, J. Yao, and Y. Cui, Nano Lett. 13, 1341 (2013)

[37] K. Kaneko, K. Inoke, K. Sato, K. Kitawaki, H. Higashida, I. Arslan, and P.A. Midgley, Ultramicroscopy 108, 210 (2008)

[38] J.S. Barnard, J. Sharp, J.R. Tong, and P.A. Midgley, Science 313, 319 (2006)

[39] T. Schwarz, O. Cojocaru-Mirédin, P. Choi, M. Mousel, A. Redinger, S. Siebentritt, and D. Raabe, J. Appl. Physics 118, 095302 (2015)

[40] A.D. Giddings, J.G. Keizer, M. Hara, G.J. Hamhuis, H. Yuasa, H. Fukuzawa, and P.M. Koenraad, Phys. Rev. B 83, 205308 (2011)

[41] L. Rigutti, B. Bonef, J. Speck, F.Tang, and R.A. Oliver, Scripta Materialia 148, 75 (2018)

[42] F.C-P. Massabuau, P.H. Griffin, H.P. Springbett, Y. Liu, R. Vasant Kumar, T. Zhu, and R.A. Oliver, APL Mater. 8, 031115 (2020)

[43] P.H. Griffin, K.M. Patel, T. Zhu, R.M. Langford, V.S. Kamboj, D.A. Ritchie, and R.A. Oliver, J. Appl. Phys. 127, 193101 (2020)

[44] R.A. Oliver, G.A.D. Briggs, M.J. Kappers, C.J. Humphreys, S. Yasin, J.H. Rice, J.D. Smith, and R.A. Taylor, Appl. Phys. Lett. 83, 755 (2003)

[45] T. Zhu, Y. Liu, T. Ding, W.Y. Fu, J. Jarman, C.X. Ren, R. Vasant Kumar, and R.A. Oliver, Sci. Rep. 7, 45344 (2017)

[46] G. Cliff, and G.W. Lorimer, J. Microsc. 103, 203 (1975) 
Sequential plan-view imaging of sub-surface structures in the transmission electron microscope

F.C-P. Massabuau ${ }^{1,2}$, H.P. Springbett ${ }^{1}$, G. Divitini ${ }^{1}$, P.H. Griffin ${ }^{1}$, T. Zhu ${ }^{1}$, R.A. Oliver ${ }^{1}$

${ }^{1}$ Department of Materials Science and Metallurgy, University of Cambridge, Cambridge CB3 OFS, UK

${ }^{2}$ Department of Physics, SUPA, University of Strathclyde, Glasgow G4 ONG, UK

E-mail: f.massabuau@strath.ac.uk 

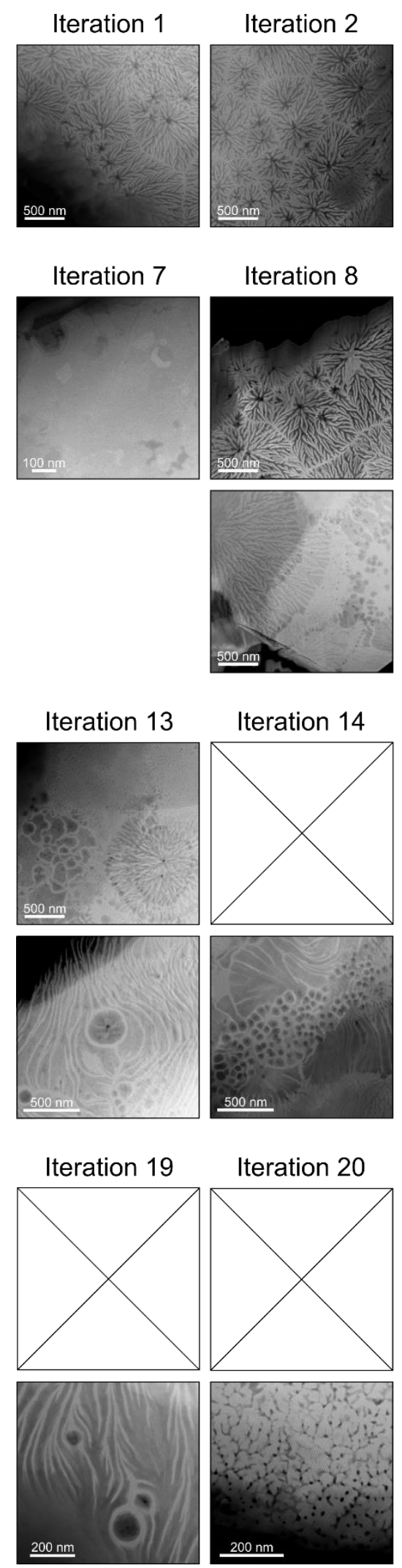
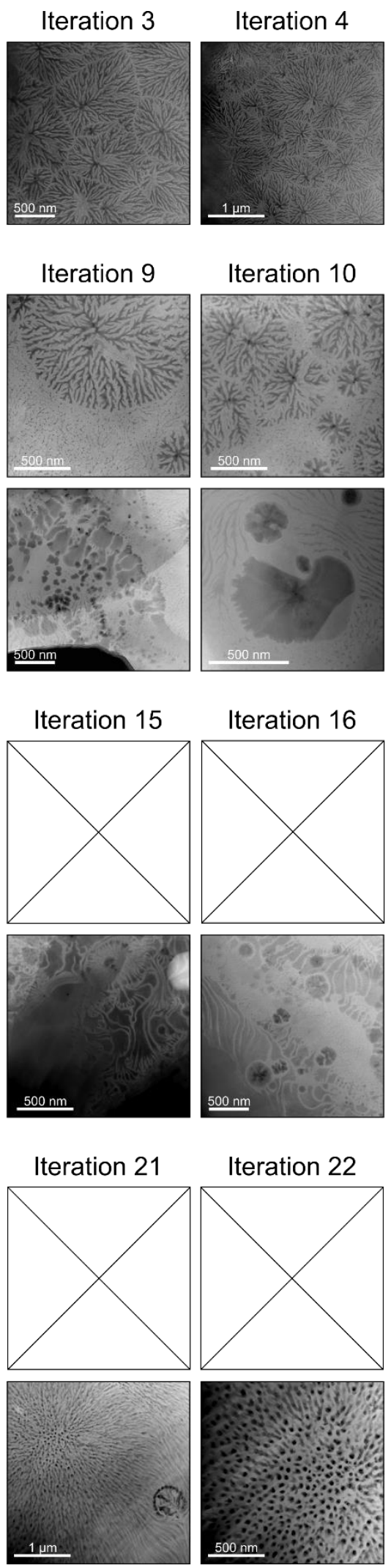

\begin{abstract}
Iteration 16
\end{abstract}
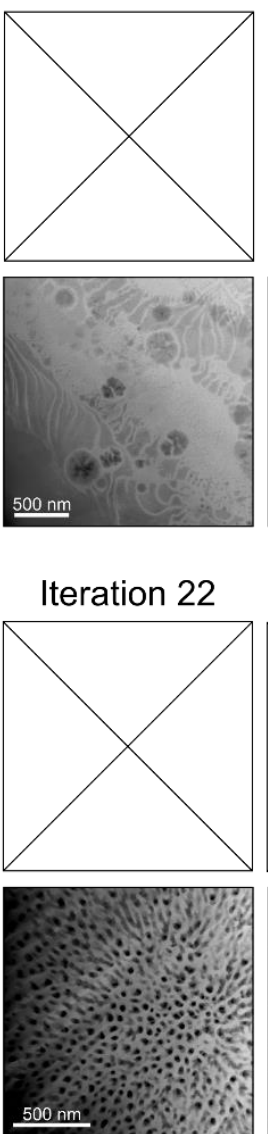
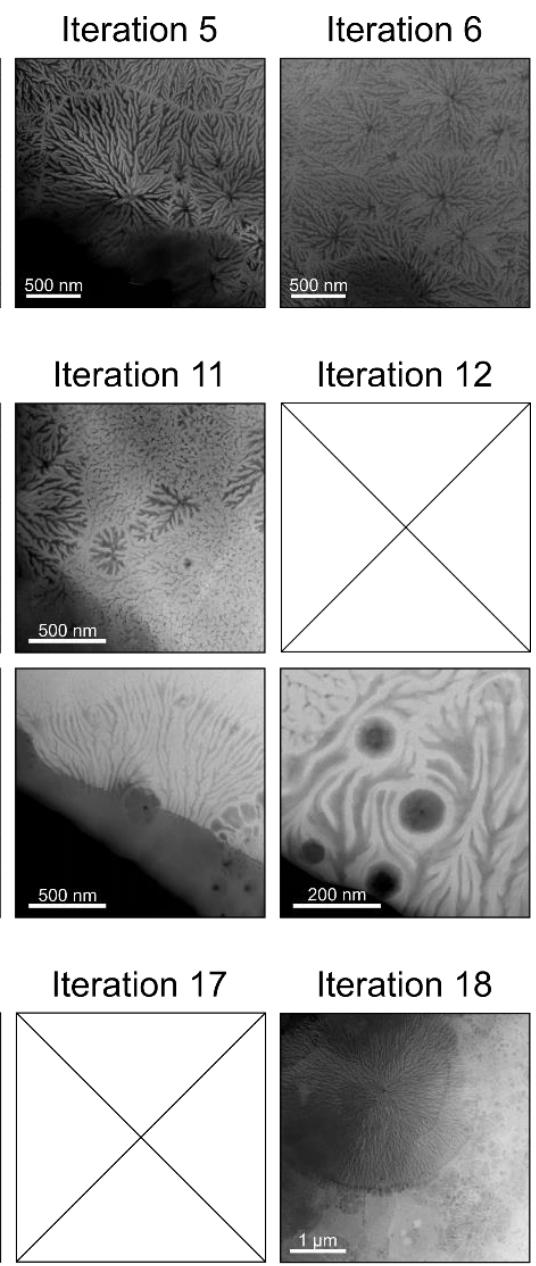

Iteration 18
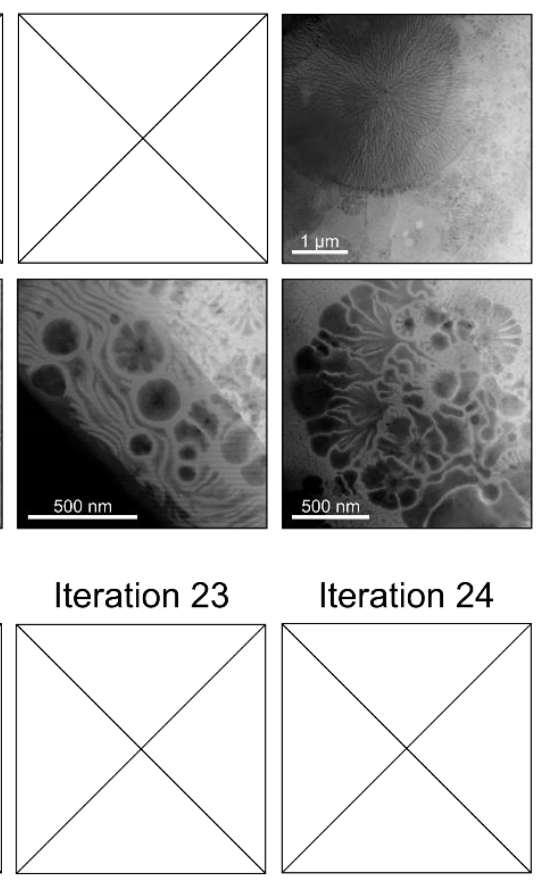

Iteration 24
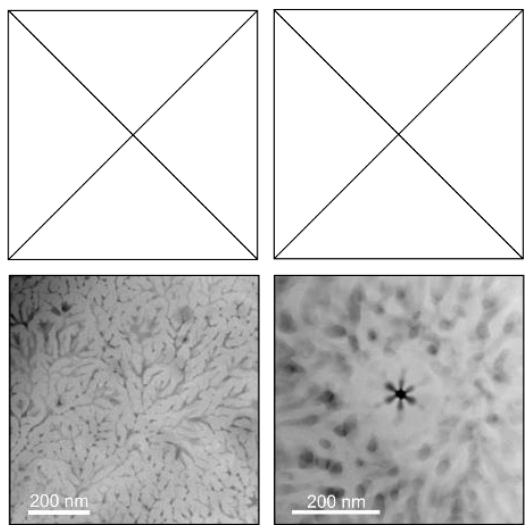

Figure S1-Montage of HAADF-STEM images from iterations 1 to 24. For each iteration, the top image highlights the porous domains (crossed squares mean no data is available), and the bottom depicts (an example of) alternative porous structures. Iterations 1-6: Top DBR, solely porous domains; Iteration 7: InGaN quantum dot layer; Iterations 8-24: Bottom DBR, porous domains + alternative structures. 